\title{
PURE STATES AND APPROXIMATE IDENTITIES ${ }^{1}$
}

\author{
JOHAN F. AARNES AND RICHARD V. KADISON
}

1. Introduction. In this note we show that each norm separable $C^{*}$-algebra has an increasing abelian approximate identity and that, if the algebra has an identity, each pure state is multiplicative on some maximal abelian subalgebra.

Let $A$ be a $C^{*}$-algebra without an identity element. A net $\left\{u_{i}\right\}_{i \in I}$ $\subseteq A$, where $I$ is a directed index set, is called an approximate identity for $A$ if $\left\|\dot{u}_{i}\right\| \leqq 1$ for all $i \in I$, and $\left\|u_{i} x-x\right\| \rightarrow 0 ;\left\|x u_{i}-x\right\| \rightarrow 0$ for all $x \in A$. We say that $\left\{u_{i}\right\}_{i \in I}$ is increasing if $u_{i} \geqq 0$ and $i \leqq j \Rightarrow u_{i} \leqq u_{j}$ for all $i, j \in I$. With $u_{i}$ selfadjoint, if one of the limits exists, so does the other. Each $C^{*}$-algebra has an increasing approximate identity $[2,1.7 .2]$. An approximate identity $\left\{u_{i}\right\}_{i \in I}$ is countable if $I$ is countable. It is abelian if $u_{i}$ and $u_{j}$ commute for all $i, j \in I$.

An element $x \in A$ is said to be strictly positive if $\rho(x)>0$ for each nonzero positive linear functional $\rho$ on $A$. A strictly positive element is positive $[2,2.6 .2]$.

We use the following notation: If $M$ is a collection of vectors in a Hilbert space $H$, and $\mathcal{F}$ is a family of bounded linear operators on $H$, then $[\mathfrak{F} M]$ is the closed linear span of the set $\{F \xi: F \in \mathcal{F}, \xi \in M\}$.

\section{Results.}

Lemma 1. If $x \in A$ is strictly positive, and $\pi$ is a nondegenerate representation of $A$ on a Hilbert space $H$, then $[\pi(x) H]=H$.

Proof. Suppose $0 \neq \xi \in[\pi(x) H]^{\perp}$. Since $\pi$ is nondegenerate there is an element $a \in A$ such that $\pi(a) \xi \neq 0$. Let $\rho=\omega_{\xi} \circ \pi$, where $\omega_{\xi}$ is the positive linear functional $y \rightarrow(y \xi, \xi)$. Then $\rho(a * a)=(\pi(a * a) \xi, \xi)$ $=\|\pi(a) \xi\|^{2} \neq 0$, so $\rho$ is a positive, nonzero linear functional on $A$. But $\rho(x)=(\pi(x) \xi, \xi)=0$ which contradicts the assumption that $x$ is strictly positive. Hence $[\pi(x) H]^{\perp}=(0)$ and the lemma follows.

Theorem 1. A $C^{*}$-algebra $A$ has a countable increasing abelian approximate identity if and only if $A$ contains a strictly positive element.

Proof. If $x_{0} \in A$ is strictly positive, we may take $x_{0}$ with norm equal to 1 . Let $u_{i}=x_{0}^{1 / t}$, and observe that $u_{i} \geqq 0,\left\|u_{i}\right\|=1 ; i \geqq j \Rightarrow u_{i} \geqq u_{j}$ and $u_{i}$ and $u_{j}$ commute for all $i, j \in I$. We want to show that for any

Received by the editors July 18, 1968.

${ }^{1}$ Research supported in part by NSF GP-7683. 
$x \in A,\left\|x u_{i}-x\right\| \rightarrow 0$. It is sufficient to do this for $x \geqq 0$. (Using Theorem 5.1 of Akemann [1], the present proof could be somewhat shortened, but we prefer to give a complete argument.)

Let $x \geqq 0$, and let $y$ be the unique positive square root of $x$. Let $\tilde{A}$ be the $C^{*}$-algebra obtained by adjoining an identity $e$ to $A$. Then $u_{i} \leqq e$ for all $i$, so that $0 \leqq y u_{i} y \leqq y e y=x$, and $y u_{i} y \leqq y u_{j} y$ if $i \leqq j$. Hence, if $z_{i}=x-y u_{i} y,\left\{z_{i}\right\}$ becomes a monotone decreasing sequence of positive elements in $A$. We claim that $\left\|z_{i}\right\| \rightarrow 0$. Let

$$
S=\left\{\rho \in A^{*}: \rho \geqq 0 ;\left\|\rho_{\|}\right\| \leqq 1\right\} .
$$

$S$ is compact in the $w^{*}$-topology $[2,2.5 .5]$. We may regard each $z_{i}$ as a continuous function on $S$ by the evaluation map. Since $z_{i} \geqq 0,\left\|z_{i}\right\|$ $=\sup \left\{\rho\left(z_{i}\right): \rho \in S\right\}[2,2.7 .3]$; so that it suffices to show that $z_{i}$ converges uniformly to 0 on $S$. As the sequence $z_{i}$ is monotone, this will follow from Dini's theorem once we know that $\rho\left(z_{i}\right) \rightarrow 0$ for each $\rho \in S$.

Let $\pi$ be a nondegenerate representation of $A$ on a Hilbert space $H$. $\pi\left(u_{i}\right)=\pi\left(x_{0}^{1 / i}\right)=\pi\left(x_{0}\right)^{1 / i}$, which by spectral theory converges strongly to the range projection of $\pi\left(x_{0}\right)$. Since $x_{0}$ is strictly positive it follows by Lemma 1 that $\pi\left(u_{i}\right) \rightarrow I$ strongly on $H$, where $I$ is the identity operator on $H$.

Let $\rho \neq 0$ be an arbitrary element of $S$ and $\pi_{\rho}$ be the associated representation of $A$ on the Hilbert space $H_{\rho}$. Then $\pi_{\rho}$ is nondegenerate with a cyclic vector $\xi_{\rho}$ and

$$
\begin{aligned}
\rho\left(z_{i}\right) & =\left(\pi_{\rho}\left(z_{i}\right) \xi_{\rho}, \xi_{\rho}\right) \\
& =\left(\left(\pi_{\rho}(x)-\pi_{\rho}\left(y u_{i} y\right)\right) \xi_{\rho}, \xi_{\rho}\right) \\
& =\left(\pi_{\rho}(y) \xi_{\rho}-\pi_{\rho}\left(u_{i}\right) \pi_{\rho}(y) \xi_{\rho}, \pi_{\rho}(y) \xi_{\rho}\right)
\end{aligned}
$$

which converges to zero since $\pi_{\rho}\left(u_{i}\right) \rightarrow I$ strongly. Hence $\left\|z_{i}\right\| \rightarrow 0$.

Working in $\tilde{A}$ (as Akemann does in [1]), let $v_{i}$ be the positive square root of $e-u_{i}$. Then $\left\|y v_{i}\right\|^{2}=\left\|y v_{i} v_{i} y\right\|=\left\|y\left(e-u_{i}\right) y\right\|=\left\|x-y u_{i} y\right\|$ $\rightarrow 0$, and hence $\left\|x u_{i}-x\right\|=\left\|y^{2} v_{i}^{2}\right\| \leqq\|y\| \cdot\left\|y v_{i}\right\| \cdot\left\|v_{i}\right\| \leqq\|y\| \cdot\left\|y v_{i}\right\| \rightarrow 0$. Thus $\left\{u_{i}\right\}$ is an approximate identity.

Conversely, suppose $\left\{u_{i}\right\}$ is an increasing abelian approximate identity, and let $x=\sum_{n=1}^{\infty} 2^{-n} u_{n}$. If $\rho$ is a nonzero positive linear functional on $A$, we know that $\rho\left(u_{n}\right) \rightarrow\|\rho\|[2,2.1 .5]$. Hence $\rho\left(u_{n}\right)>0$ for some $n$, so $\rho(x)=\sum_{n=1}^{\infty} 2^{-n} \rho\left(u_{n}\right)>0$. This shows that $x$ is strictly positive, and the proof is complete.

Observe that if $A$ is separable, then $A$ has a strictly positive element. Indeed, if $\left\{y_{n}\right\}$ is dense in $A$, then $\left\{x_{n}=y_{n}^{*} y_{n}\right\}$ is dense in $A^{+}=\{x \in A: x \geqq 0\}$. Clearly $x=\sum_{n=1}^{\infty}\left(2^{n}\left\|x_{n}\right\|\right)^{-1} x_{n}$ is strictly positive in $A$. 
Corollary 1. Any separable $C^{*}$-algebra has a countable increasing abelian approximate identity.

REMARK. Let $X$ be a locally compact Hausdorff space, $A=\mathrm{e}^{0}(X)$, the $C^{*}$-algebra of all continuous complex functions on $X$ vanishing at infinity. It is easily verified that $A$ contains a function $f$ which is everywhere positive if and only if $X$ is $\sigma$-compact. Since each state on $A$ may be represented by a Borel measure on $X$, we see that such a function $f$ is a strictly positive element of $A$. Evidently $X$ may be $\sigma$-compact without having a countable base for its topology, so $A$ may have a strictly positive element without being separable. Needless to say, $A$ will not always have strictly positive elements. An example is $\mathrm{C}^{0}(R)$, when $R$ is given the discrete topology.

A positive linear functional $\rho$ on a $C^{*}$-algebra $A$ is a state if $\|\rho\|=1$. If $A$ has an identity $e$, this is equivalent to the condition $\rho(e)=1$. We say $\rho$ is pure if $\rho \neq 0$ and each positive, linear functional $\gamma$ on $A$ such that $0 \leqq \gamma \leqq \rho$, is of the form $\gamma=a \rho ; 0 \leqq a \leqq 1$.

Theorem 2. Let $A$ be a separable $C^{*}$-algebra with identity. If $\rho$ is a pure state on $A$, then there is a maximal abelian $C^{*}$-subalgebra $B$ of $A$ such that $\rho \mid B$ is multiplicative.

Proof. Let $N_{\rho}$ be $\{x \in A: \rho(x * x)=0\}$ and $A_{0}$ be $N_{\rho} \cap N_{\rho}^{*} . A_{0}$ is a $C^{*}$-subalgebra of $A$ and is therefore separable. Hence $A_{0}$ contains a strictly positive element $x_{0}$. Let $B_{0}$ be a maximal abelian $C^{*}$-subalgebra of $A_{0}$ containing $x_{0}$ and $B$ be $B_{0}+C \cdot e$. Then $B$ is an abelian $C^{*}$-subalgebra of $A$. Since $\rho$ vanishes on $B_{0}, \rho \mid B$ is multiplicative of norm 1. To show that $B$ is a maximal abelian $C^{*}$-subalgebra of $A$, it suffices to show that if a selfadjoint $x$ in $A$ commutes with $B$, then $x \in B$. Now, $x \in B$ if and only if $x-\rho(x) e \in B$; so we may assume that $\rho(x)=0$. Let $\pi_{\rho}$ be the irreducible representation of $A$ associated with $\rho$ on the Hilbert space $H_{\rho}$, with cyclic vector $\xi_{\rho}[2,2.5 .4]$. Let $H_{0}$ $=\left[\xi_{\rho}\right]^{\perp}$. We claim that $\left[\pi_{\rho}\left(A_{0}\right) H_{\rho}\right]=H_{0}$. Indeed, if $y \in A_{0}$ then $\left\|\pi_{\rho}(y) \xi_{\rho}\right\|^{2}=\left(\pi_{\rho}(y * y) \xi_{\rho}, \xi_{\rho}\right)=\rho(y * y)=0$; so that $\left(\pi_{\rho}(y) \pi_{\rho}(x) \xi_{\rho}, \xi_{\rho}\right)=0$ for all $x$ in $A$. On the other hand, let $\xi$ in $H_{0}$ be arbitrary. By the transitivity theorem $[2,2.8 .3]$ there is a selfadjoint element $y \in A$ such that $\pi_{\rho}(y) \xi_{\rho}=0$ and $\pi_{\rho}(y) \xi=\xi$. But then $y \in A_{0}$ and the claim follows. Hence $\pi_{\rho} \mid A_{0}$ is a nondegenerate representation on $H_{0}$. Let $E$ be the orthogonal projection of $H_{\rho}$ onto $H_{0}$. By Lemma $1,\left[\pi_{\rho}\left(x_{0}\right) H_{0}\right]$ $=H_{0}$. Now $x_{0}$ and $x$ commute; so that $E$ and $\pi_{\rho}(x)$ commute. Hence $H_{0}$ and $\left[\xi_{\rho}\right]$ are invariant under $\pi_{\rho}(x)$. This means that $\xi_{\rho}$ is an eigenvector for $\pi_{\rho}(x)$; so $\pi_{\rho}(x) \xi_{\rho}=a \xi_{\rho}$ for some real $a$. Now $0=\rho(x)$ $=\left(\pi_{\rho}(x) \xi_{\rho}, \xi_{\rho}\right)=a\left(\xi_{\rho}, \xi_{\rho}\right)=a$. Hence $\pi_{\rho}(x) \xi_{\rho}=0$ so $x \in A_{0}$. Since $B_{0}$ is 
maximal abelian in $A_{0}$ it follows that $x \in B_{0} \subseteq B$. The proof is complete.

\section{REFERENCES}

1. C. Akemann, Interpolation in $W^{*}$-algebras, Duke Math. J. 35 (1968), 525-534.

2. J. Dixmier, Les $C^{*}$-algebres et leurs representations, Gauthier-Villars, Paris, 1964.

UNIVERSITY OF OSLO AND

University of Pennsylvania 